\title{
PLASMA-SURFACE INTERACTIONS UNDER HIGH HEAT AND PARTICLE FLUXES
}

\author{
Gregory De Temmerman ${ }^{a} *$, Kirill Bystrov ${ }^{a}$, Feng LiU $^{b}$, Wei LiU ${ }^{c}$, \\ Thomas Morgan ${ }^{a}$, Irem Tanyeli ${ }^{a}$, Miranda van den Berg ${ }^{a}$, Haiyan Xu ${ }^{c}$, \\ JAKUB ZIELINSKI ${ }^{a}$
}

${ }^{a}$ FOM Institute DIFFER, Dutch Institute For Fundamental Energy Research, Edisonbaan 14, 3439 MN Nieuwegein, The Netherlands

${ }^{b}$ Science and Technology on Marine Corrosion and Protection laboratory, Luoyang Ship Material Research Institute, Qingdao 266101, P.R. China

${ }^{c}$ Department of materials science and engineering, Tsinghua University, Beijing, 100084, P.R. China

* corresponding author: g.c.detemmerman@differ.nl

\begin{abstract}
The plasma-surface interactions expected in the divertor of a future fusion reactor are characterized by extreme heat and particle fluxes interacting with the plasma-facing surfaces. Powerful linear plasma generators are used to reproduce the expected plasma conditions and allow plasma-surface interactions studies under those very harsh conditions. While the ion energies on the divertor surfaces of a fusion device are comparable to those used in various plasma-assited deposition and etching techniques, the ion (and energy) fluxes are up to four orders of magnitude higher. This large upscale in particle flux maintains the surface under highly non-equilibrium conditions and bring new effects to light, some of which will be described in this paper.
\end{abstract}

KEYWORDS: plasma-surface interactions, refractory metals, carbon, linear plasma generator.

\section{INTRODUCTION}

In a magnetic confinement fusion device, power from the core plasma has to be exhausted by the plasmafacing components, mainly in the divertor area, a special area of the plasma chamber, where the open magnetic field lines intersect the primary plasma facing components and where the plasma is neutralized and pumped away [22]. In ITER, the steadystate heat load onto the divertor plates will be about $10 \mathrm{MW} \mathrm{m}^{-2}[23$. In addition, the very high localized heat fluxes caused by so-called Edge Localized Modes (several $\mathrm{GW} \mathrm{m}^{-2}$ for $0.5 \div 1 \mathrm{~ms}$ ) is high enough to lead to material erosion, melting and vaporization for most materials, and represent a serious concern for the lifetime of the plasma-facing components, because of the expected ELM frequency of up to $40 \mathrm{~Hz}$. In parallel, the divertor surfaces will be exposed to intense particle fluxes (up to $10^{24} \mathrm{~m}^{-2} \mathrm{~s}^{-1}$ or $1.6 \times 10^{5} \mathrm{~A} \mathrm{~m}^{-2}$ ) with energies below $50 \mathrm{eV}$, for extended durations of times [18. Those conditions cannot be reproduced in present fusion devices, and powerful linear plasma generators have been developed to allow the study of plasma-surface interactions under those extreme conditions of heat and particles.

This paper will describe the high flux facilities available at the FOM Institute DIFFER and give a brief overview of the specifics of plasma-surface interactions under the uniquely achievable high heat and particle fluxes.

\section{High FLUX PLASMA GENERATORS}

Linear plasma generators, such as the PISCES facilities at UC San Diego [10, 11] or the NAGDIS facilities at the University of Nagoya [21, have long been used for the study of plasma-surface interaction under fusion-relevant conditions. The achievable ion flux density in those devices is typically limited to $1 \times 10^{23} \mathrm{~m}^{-2} \mathrm{~s}^{-1}$ in steady-state, a factor of 10 lower than what is expected in the divertor of ITER. In addition, it is currently not possible to generate transient plasma pulses in those devices and, instead, high power lasers are used to combine a plasma environment and transient heat fluxes and get some insights into the effects of ELMs on plasma-exposed surface [13, 28, albeit missing the transient particle flux associated with an ELM. In parallel, powerful plasma guns [9] can be used to study the effect of powerful transient plasma pulses on surfaces, in the absence of continuous plasma loading however.

In order to fill the existing gaps and allow material exposure to ion fluxes higher than $10^{24} \mathrm{~m}^{-2} \mathrm{~s}^{-1}$, the Pilot-PSI and Magnum-PSI devices were developed at FOM DIFFER.

A schematic diagram of Pilot-PSI is shown in Fig. 1. Plasma is generated by a so-called cascaded arc source [29] and exhausts into the vacuum vessel. An axial magnetic field of up to $1.6 \mathrm{~T}$ is used to confine the plasma and generate an intense magnetized cylindrical plasma beam. The maximum pulse duration is dependent on the magnetic field strength and is typically $100 \mathrm{~s}$ at $0.4 \mathrm{~T}$ and $10 \mathrm{~s}$ at $1.6 \mathrm{~T}$. Discharge 


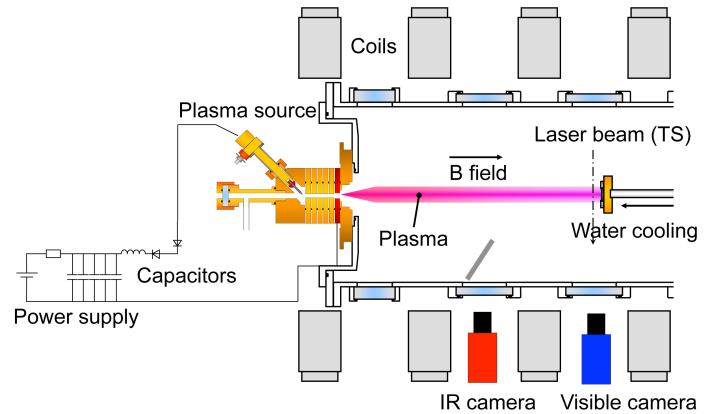

Figure 1. Schematic diagram of the Pilot-PSI linear plasma device.

currents in the range of $150 \div 250 \mathrm{~A}$ and gas flows in the range of $1 \div 3 \mathrm{slm}$ ( 1 standard litre per minute is $4.48 \times 10^{20}$ part $^{-1}$ ) are typically used. Pumping of the process gas $(\mathrm{H}, \mathrm{D}, \mathrm{He}, \mathrm{Ne}, \mathrm{N}, \mathrm{Ar})$ is ensured by mechanical booster pumps with an effective pumping capacity of $7200 \mathrm{~m}^{3} / \mathrm{hr}$. The samples are clamped on a water-cooled copper holder, an intermediate layer of Grafoil is used to improve the thermal contact, and are located at a disctance of $54 \mathrm{~cm}$ from the plasma source. In addition, the device offers the unique possibility to combine the continuous plasma with a transient heat and particle pulse (up to $1.2 \mathrm{GW} \mathrm{m}^{-2}$ for $1 \mathrm{~ms}$ ) allowing the study of ELM effects on plasmaexposed surfaces [6, 7]. The ELM-like pulses are generated by discharging a capacitor bank system into the plasma source to transiently increase the input power, and thus the plasma density and temperature. Figure 2 gives an overview of the plasma conditions, for both continuous and pulsed plasma, measured by Thomson scattering at a distance of $17 \mathrm{~mm}$ from the exposed surface. For DC plasmas, the densities of up $2 \div 3 \times 10^{21} \mathrm{~m}^{-3}$ with temperatures below $3 \mathrm{eV}$ have been attained. Pulsing the plasma source results in a strong increase of both the plasma densities (up to $10^{22} \mathrm{~m}^{-3}$ ) and temperatures (up to $20 \mathrm{eV}$ ).

Pilot-PSI served as a development platform for the Magnum-PSI device which is a large linear plasma generator designed for steady-state operations. Magnum-PSI is designed around a $2.5 \mathrm{~T} \mathrm{su}-$ perconducting coil, the same cascaded arc source used on Pilot-PSI, and a large pumping capability of about $53000 \mathrm{~m}^{3} / \mathrm{hr}$. The device is $15 \mathrm{~m}$ long. A sophisticated target system has been developed in order to accommodate a wide variety of sample size and shapes, from small disc-type samples to targets with dimensions up to $60 \times 12 \mathrm{~cm}$ and $100 \mathrm{~kg}$ [3. The target head is attached to the target manipulator allowing the withdrawal of the target from its exposure position to the target exchange chamber. This target exchange chamber can be isolated from the main vacuum chamber with its exposure chamber via a double gate valve system. This allows for an in-situ transfer of the target from the exposure location to the target exchange chamber in less than $30 \mathrm{~s}$ in the presence of the magnetic field. The machine is currently operated with

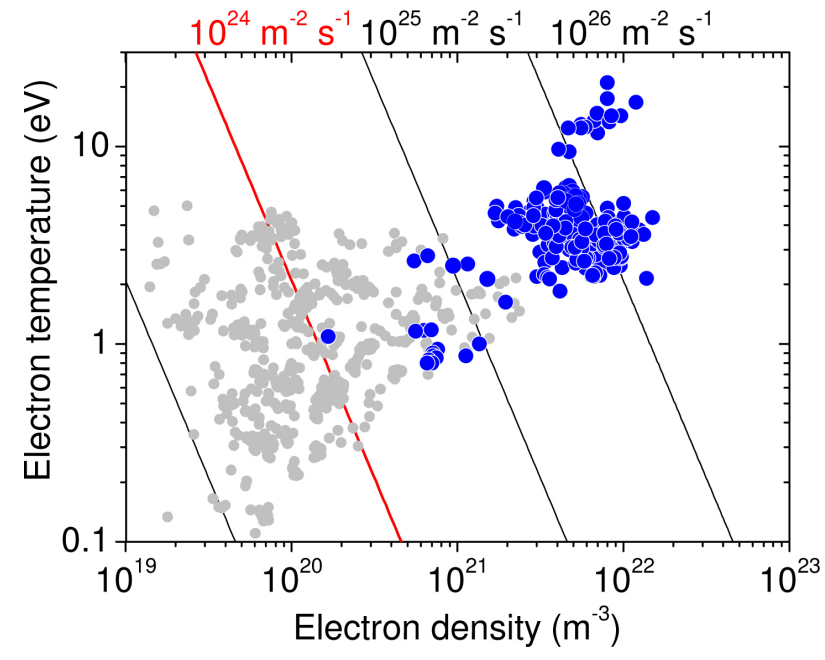

FiguRe 2. Illustration of the achievable plasma conditions in Pilot-PSI for various settings of the magnetic field, gas flow and input power; the dark grey points indicate the DC plasma conditions, while the blue points denote the plasma conditions during high power pulses.
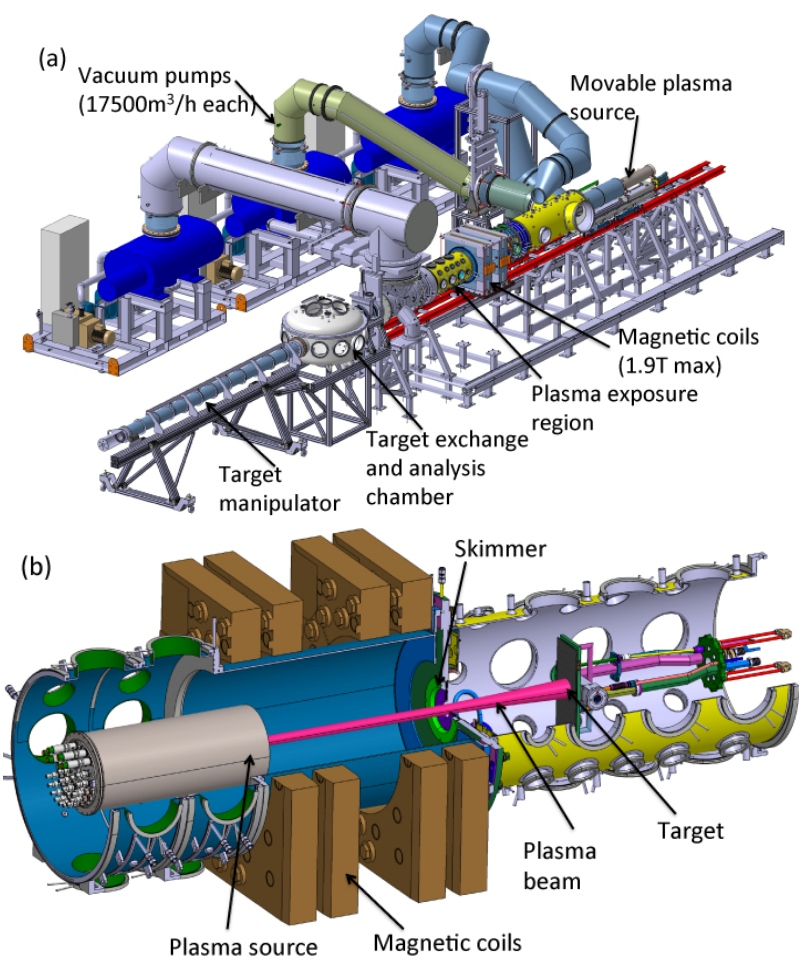

FiguRE 3. Schematic diagram of the Magnum-PSI linear plasma device: (a) global view on the device with a description of the main components, (b) cut-out view through the vacuum vessel illustrating the geometry of the magnetic coil arrangement as well as a typical plasma exposure.

conventional copper coils generating a magnetic field of up to $1.9 \mathrm{~T}$ for $7 \mathrm{~s}$ (the lower the field the longer the discharge), allowing similar plasma conditions as in Pilot-PSI albeit with a larger plasma beam diameter. A schematic diagram of Magnum-PSI in its present configuration is shown in Fig. 3 . 


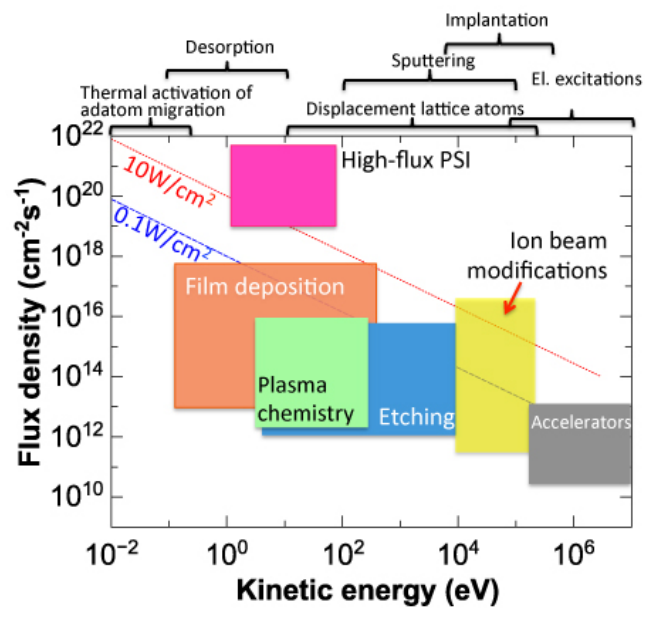

Figure 4. Typical operation windows for various ion-induced processing techniques; also indicated are the typical conditions used during the experiments described in this work.

\section{PSI UNDER HIGH HEAT AND PARTICLE FLUXES}

\subsection{FluX: HOW HIGH IS High?}

The linear plasma devices at FOM can generate plasma fluxes of up to $10^{25} \mathrm{~m}^{-2} \mathrm{~s}^{-1}$ in DC operations and up to $10^{26} \mathrm{~m}^{-2} \mathrm{~s}^{-1}$ for ms durations when the plasma source is pulsed. The energy of the ions impinging on the surface is determined by their acceleration in the sheath region. When the target is left floating, and since the electron temperature is typically lower than $3 \mathrm{eV}$ (except during plasma pulses), the ion energy remains lower than $15 \mathrm{eV}$ - assuming $T_{\mathrm{e}}=T_{\mathrm{i}}$. The ion energy can be varied by negative biasing of the sample with respect to the plasma potential. The negative bias voltage is at maximum $100 \mathrm{~V}$, with arcing occuring for higher voltages. Before discussing how such intense fluxes of low energy ions interact with surfaces, it is of primary interest to define the flux magnitude by comparison with that typically encountered in other plasma processing technologies. Figure 4 summarizes typical values of ion energy and ion fluxes to the surface in various plasma-assisted processes. While the ion energies used in this study (and in the divertor of a fusion device) are comparable to those used in various plasma-assited deposition and etching techniques, the ion (and energy) fluxes are several (up to four) orders in a magnitude higher. Some effects related to this large upscale in particle flux will be described in the next sections.

\subsection{PARTiCle RECYCling}

As mentioned above, the plasmas used in our studies are characterized with a low electron temperature and a high plasma density. Under those conditions, the mean free path of particles released from the surface is very short and typically shorter than the characteristic size of the plasma. In the case of the chemical

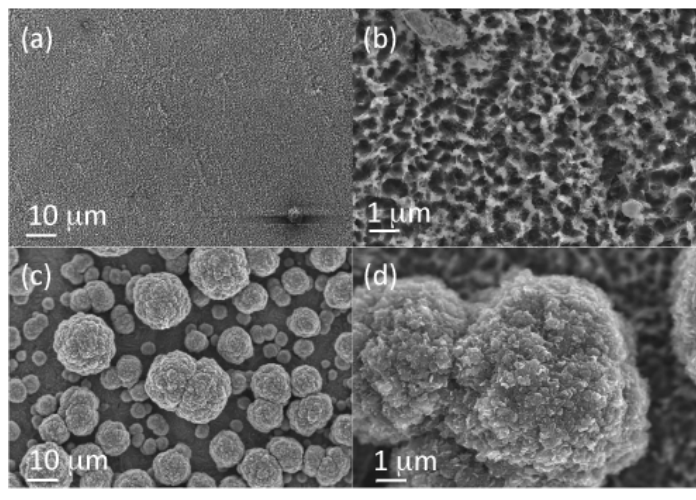

FiguRE 5. Characteristic SEM images of a graphite surface after plasma exposure in case of negligible re-deposition ( $\mathrm{a}$ and $\mathrm{b}$ ) and a surface covered with cauliflower-like dust structures (c and d).

sputtering of graphite by hydrogen plasmas, for example, for $0.3 \mathrm{eV} \leq T_{\mathrm{e}} \leq 2 \mathrm{eV}$, the ionization of the hydrocarbons released from the surface primarily occurs through charge exchange. The ionization mean-free path strongly depends on the plasma density in front of the surface and for instance decreases from $3.2 \mathrm{~mm}$ for $n_{\mathrm{e}}=1 \times 10^{20} \mathrm{~m}^{-3}$ to $0.8 \mathrm{~mm}$ for $n_{\mathrm{e}}=4 \times 10^{20} \mathrm{~m}^{-3}$. This has to be compared with the Full-Width at Half Maximum of the plasma beam which is about $10 \mathrm{~mm}$. As a consequence, every eroded particle will experience a cycle of erosion/re-deposition events before it can eventually escape the plasma beam. Modeling shows that every $\mathrm{CH}$ molecule eroded from the surface, for a density of $4 \times 10^{20} \mathrm{~m}^{-3}$, will visit the surface in average 19 times before actually escaping the plasma beam [26]. As a result, this strong material recycling reduces the net surface erosion and it has been experimentally determined that up to $90 \%$ of the eroded material is re-deposited back on the surface [4]. Redeposits appear in the form of large cauliflower particles which cover the surface, the surface coverage depends on the re-deposition fraction.

\subsection{SuRfaCE SELF-ORGANIZATION}

For plasma fluxes higher than $10^{24} \mathrm{~m}^{-2} \mathrm{~s}^{-1}$, each surface site will be visited every $10 \mu$ or less, time which is shorter than the residence time of adsorbed particles and in the range of typical relaxation times for processes such as diffusion of vacancies and interstitials, phase transformation and chemical reactions. In addition, low-energy ions $(\leq 10 \mathrm{eV})$, having kinetic energies in the range of the interatomic binding energies, can transfer their energies very efficiently to surface atoms thus enhancing adatoms mobility, leading to enhanced surface diffusion and reactivity for example. In other words, incoming ions will interact with a surface, which is far out of equilibrium. Those conditions promote self-organization effects and the appearance of novel structures.

A striking example of ion-induced surface selforganization was obtained when studying the chemical sputtering of a fine grain graphite by a high 


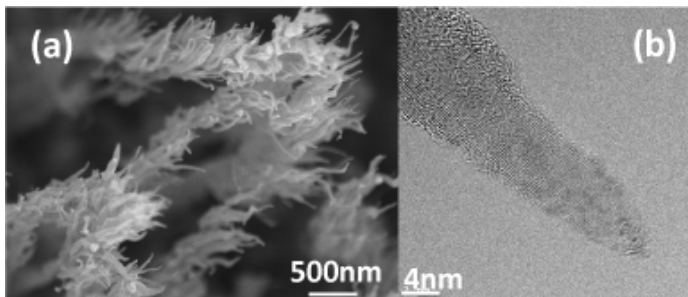

Figure 6. (a) Secondary and (b) Transmission Electron Microscopy images of carbon nanotubes formed during the interaction between a high flux nitrogen plasma and a polished fine grain graphite substrate.

flux nitrogen plasma. Injection of extrinsic impurities such as nitrogen might be used in ITER to decrease the power loads onto the divertor surfaces, such an effect has been succesfully demonstrated in the ASDEX-Upgrade tokamak [16]. The interaction of nitrogen ions with carbon surfaces is known to lead to the formation of volatile compounts ( $\mathrm{CN}$ for example) and has mainly been studied for low flux conditions and for energies above $50 \mathrm{eV}$ [12. Since the chemical sputtering of carbon by hydrogen is thought to be flux dependent with a pronounced decrease of the erosion yield with fluxes [25], studies were carried out in Pilot-PSI to investigate the chemical sputtering by high fluxes of nitrogen ions. Polished polycrystalline graphite samples were exposed to high-density magnetized nitrogen plasmas $\left(n_{\mathrm{e}}=1.5 \times 10^{20} \mathrm{~m}^{-3}, T_{\mathrm{e}}=1 \mathrm{eV}\right)$, with ion energy of $30 \mathrm{eV}$. After $150 \mathrm{~s}$ of plasma exposure, at a surface temperature of $900^{\circ} \mathrm{C}$ (the temperature depends on the plasma-deposited power), the formation of carbon nanotubes was observed on the surface by highresolution microscopy (Fig. 6). The nanotubes length is typically lower than $500 \mathrm{~nm}$ and their diameter around $5 \div 10 \mathrm{~nm}$. A transmission electron microscopy (Fig. 6b) confirms that the nanotubes are multi-wall and also indicate the presence of amorphous carbon around the tubed. It should be mentioned that no special pre-treatment of the surface was applied and that the nanotubes grew uniquely thanks to the action of the high flux plasma.

\subsection{Plasma-INDUCED MORPHOLOGY CHANGES}

Refractory metals, such as tungsten, are prime candidate for plasma-facing materials in future fusion devices due to their high melting point, good thermal properties and low sputtering yield by the low-Z species characteristic of a tokamak divertor plasma. The solubility of species like hydrogen and helium in refractory metals is very low [5]. In addition, the incoming particle flux is high enough to cause a local supersaturation of mobile gas particles within the implantation zone and strong surface morphology changes as a result of cluster/bubble growth. For example, it has recently been reported that irradiation of metal surfaces at elevated temperatures by low-energy helium ions

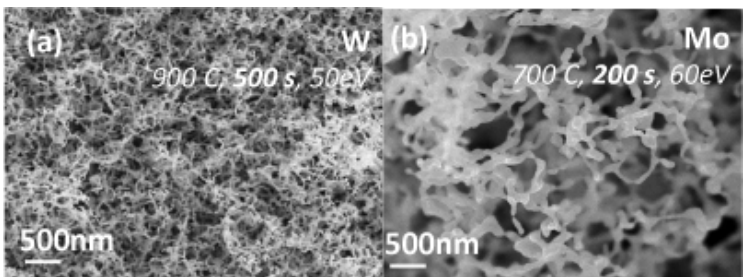

FIGURE 7. High-resolution SEM images of heliuminduced nanostructures formed on (a) tungsten and (b) molybdenum surfaces after high flux plasma exposure.

(with energy below the ion damage threshold) leads to the formation of a fibreform nano-structure, with filament diameter below $20 \mathrm{~nm}$. Figure 7 illustrates the helium-induced nanostructure formed on both tungsten and molybdenum surfaces exposed to pure helium plasmas in Pilot-PSI [8]. The conditions necessary to induce the formation of such nanostructures include a sufficiently high surface temperature, the value of which depends on the material under consideration, high enough flux of helium ions and ion energy higher than $20 \div 30 \mathrm{eV}$ [14, 1, 17]. The helium-induced nanostructures formed on tungsten and molybdenum surfaces are characterized by a very low density of less than $10 \%$ of that of the bulk material, a high level of porosity [2], and, in turn, a very low surface reflectivity making the surface appear completely black [15]. This makes the high flux plasma processing an attractive approach for the synthesis of black metals allowing maximum light absorption for solar power concentration 24 .

\subsection{SyNERGISTIC EFFECTS}

Finally, plasma instabilities, such as the so-called Edge Localized Modes (ELMs), lead to transient heat loading of the plasma-exposed surface. As mentioned above, the continuous plasma exposure leads to strong morphology changes and a high concentration of mobile gas particles in the near-surface region. This results in strong interplays between the effects of the quiescent plasma and the transient loads. It has been reported, for example, that the damage threshold of a plasma-exposed surface was significantly reduced compared to that of a virgin surface [7]. To study the role of plasma exposure on the response of the surface during transient heating in more details, tungsten targets pre-exposed to deuterium plasma in Pilot-PSI were subsequently irradiated by multiple laser pulses. The plasma exposures were made using pure deuterium plasmas (peak $n_{\mathrm{e}} e \sim 1.6 \div 3.2 \times 10^{20} \mathrm{~m}^{-3}$ and $\left.T_{\mathrm{e}} \sim 0.8 \div 1.4 \mathrm{eV}\right)$, the ion energy was fixed to $38 \mathrm{eV}$. The laser pulse spot size was about $2 \mathrm{~mm}$ diameter with the duration of $1.2 \mathrm{~ms}$ at $1064 \mathrm{~nm}$ from an Nd:YAG laser, with a repetition rate of $10 \mathrm{~Hz}$. The samples were exposed to differing fluences between $F=0.6 \times 10^{25}$ and $11.2 \times 10^{25} \mathrm{D}^{+} \mathrm{m}^{-2}$. Preexposed samples displayed clear blisters and bubbles on the surface (Fig. 87), increasing in size as a function of fluence. Each sample was then exposed to 100 pulses 


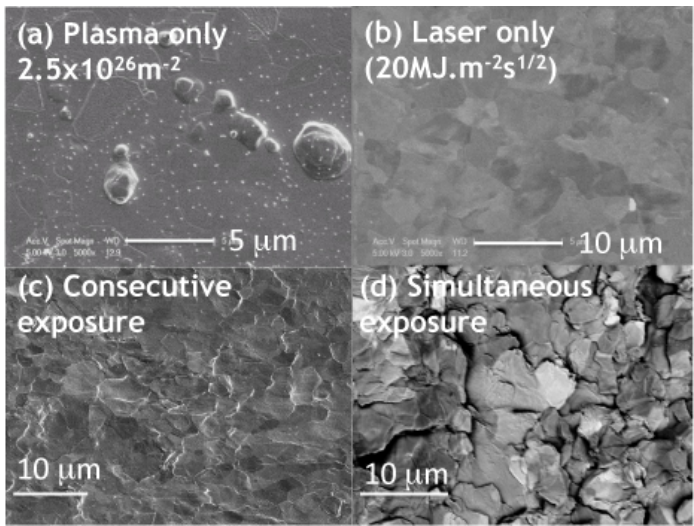

Figure 8. SEM pictures of tungsten samples exposed to either (a) only $40 \mathrm{~s}$ steady state plasma $\left(2.5 \times 10^{26} \mathrm{~m}^{-3}\right),(\mathrm{b})$ only transient laser pulses (100 $20 \mathrm{MJ} \mathrm{m}^{-2} \mathrm{~s}^{1 / 2}$ pulses) (c) consecutive exposures of (a) and (b) or (d) simultaneous exposures of 10 s steady state plasma and $100020 \mathrm{MJ} \mathrm{m}^{-2} \mathrm{~s}^{1 / 2}$ pulses.

at either 20 or $40 \mathrm{MJ} \mathrm{m}^{-2} \mathrm{~s}^{1 / 2}$. The effect of a laser exposure on a virgin surface is illustrated in Fig. 8p, where typically no surface modification can be observed. For the plasma-exposed surfaces however, after laser exposure strong surface roughening is observed for all samples (Fig. 8f). More details can be found in [20. However, no clear difference in terms of surface roughening was observed after the laser exposure between samples exposed to different plasma fluences.

In order to further test the effect of synergistic enhancement of the damage, a target was exposed to simultaneous steady state plasma and pulsed laser irradiation in Magnum-PSI. The plasma conditions were similar to those of pre-exposed targets above, while the laser produced a heat flux parameter of $20 \mathrm{MJ} \mathrm{m}^{-2} \mathrm{~s}^{1 / 2}$ for each of 1000 pulses at a $10 \mathrm{~Hz}$ repetition rate. The results (Fig. 8d) show an enhanced surface damage under those simultaneous loading conditions, with strong cracking of the surface. This further outlines the possible strong role of mobile hydrogen present in the near-surface region during the plasma exposure, and is in agreement with the results reported in [27]. Future experiments will aim at clarifying the exact mechanisms at stake and study the differences induced by the use of a pulsed plasma (which is both a source of heat and particles) and a pulsed laser.

\section{Conclusions}

The plasma-surface interactions expected in the divertor of a future fusion reactor are characterized by extreme heat and particle fluxes interacting with the plasma-facing surfaces. Those extreme conditions bring new and exciting physics posing both a serious issue for the lifetime of plasma-facing components in a reactor and an interesting glimpse into the behaviour of the surfaces under highly non-equilibrium conditions. The understanding and control of those effects is crucially needed to design materials able to withstand the harsh conditions expected in a fusion reactor. In particular, the interplays between the steady-state plasma exposure and the regular transient heat loads associated with ELMs, lead to synergistic effects resulting in a decreased damage threshold of tungsten surfaces. The impact of those effects for the lifetime of plasma-facing materials in future fusion devices needs to be further investigated. On the other hand, harnessing those extreme fluxes to manipulate material structure also opens the door to produce new structures and chemistries that are thermodynamically or kinetically inaccessible by conventional near-equilibrium techniques.

\section{ACKNowledgements}

This work was partly supported by the European Communities under the contract of Association between EURATOM/FOM and carried out within the framework of the European Fusion Programme with financial support from NWO and the NWO Grant No. RFBR 047.018.002. The views and opinions expressed herein do not necessarily reflect those of the European Commission. Financial support was also provided by the National Magnetic Confinement Fusion Science Program of China under Grant 2009GB106003 and the National Nature Science Foundation of China under contract nos. 51071095 and 50971077.

\section{REFERENCES}

[1] M. J. Baldwin, et al. The effects of high fluence mixed-species (deuterium, helium, beryllium) plasma interactions with tungsten. J Nucl Mater 390-391:886, 2009 .

[2] M. J. Baldwin, R. P. Doerner. Formation of helium induced nanostructure 'fuzz' on various tungsten grades. J Nucl Mater 404(3):165, 2010.

[3] M. A. van den Berg, et al. The target for the new plasma/wall experiment Magnum-PSI. Fus Eng Des 86(9-11):1745, 2011.

[4] K. Bystrov, et al. Reorganization of graphite surfaces into carbon micro- and nanoparticles under high flux hydrogen plasma bombardment. J Vac Sc Tech A 31(1):011303, 2013.

[5] R. A. Causey. Hydrogen isotope retention and recycling in fusion reactor plasma-facing components. $J$ Nucl Mater 300(2-3):91, 2002.

[6] G. De Temmerman, et al. Production of high transient heat and particle fluxes in a linear plasma device. Appl Phys Lett 97(8):081502, 2010.

[7] G. De Temmerman, et al. ELM simulation experiments on Pilot-PSI using simultaneous high flux plasma and transient heat/particle source. Nucl Fusion 51(7):073008, 2011.

[8] G. De Temmerman, et al. Nanostructuring of molybdenum and tungsten surfaces by low-energy helium ions. J Vac Sci Technol A 30(4):041306, 2012. 
[9] I. E. Garkusha, et al. Experimental study of plasma energy transfer and material erosion under ELM-like heat loads. J Nucl Mater 390-391:814-817, 2009.

[10] D. M. Goebel, G. Campbell, R. W. Conn. Plasma surface interaction experimental facility (PISCES) for materials and edge physics studies. J Nucl Mater 121:277, 1984.

[11] Y. Hirooka, et al. A new plasma-surface interactions research facility: PISCES-B and first materials erosion experiments on bulk-boronized graphite. J Vac Sci Technol A 8(3):1790, 1990.

[12] W. Jacob, C. Hopf, M. Schluter. Chemical sputtering of carbon by nitrogen ions. Appl Phys Lett 86(20):204103, 2005.

[13] S. Kajita, et al. Plasma-assisted laser ablation of tungsten: Reduction in ablation power threshold due to bursting of holes/bubbles. Appl Phys Lett 91(26):261501, 2007.

[14] S. Kajita, et al. Formation process of tungsten nanostructure by the exposure to helium plasma under fusion relevant plasma conditions. Nucl Fusion 49(9):095005, 2009.

[15] S. Kajita, et al. Nanostructured black metal: Novel fabrication method by use of self-growing helium bubbles. Appl Phys Express 3(8):085204, 2011.

[16] A. Kallenbach, et al. Divertor power load feedback with nitrogen seeding in ASDEX upgrade. Plasma Phys Control Fusion 52(5):055002, 2010.

[17] S. I. Krasheninnikov. Viscoelastic model of tungsten 'fuzz' growth. Phys Scr T145:014040, 2011.

[18] A. S. Kukushkin, et al. Finalizing the ITER divertor design: The key role of SOLPS modeling. Fus Eng Des 86(12):2865, 2011.

[19] S. Masuzaki, N. Ohno, S. Takamura. Experimental study on plasma heat flow to plasma-facing materials. $J$ Nucl Mater 223(3):286, 1995.
[20] T. W. Morgan. Reduced damage threshold for tungsten using combined steady state and transient sources. J Nucl Mater in press. Online, http: //dx.doi.org/10.1016/j.jnucmat.2013.01.168

[21] N. Ohno, et al. Static and dynamic behaviour of plasma detachment in the divertor simulator experiment NAGDIS-II. Nucl Fusion 41(8):1055, 2001.

[22] C. S. Pitcher, P. C. Stangeby. Experimental divertor physics. Plasma Phys Control Fusion 39(6):779, 1997.

[23] R. A. Pitts, et al. Physics basis and design of the ITER plasma-facing components. J Nucl Mater 415(1 suppl.):S957, 2011.

[24] E. Rephaeli, S. Fan. Tungsten black absorber for solar light with wide angular operation range. Appl Phys Lett 92(21):211107, 2008.

[25] J. Roth, C. Garcia-Rosales. Analytic description of the chemical erosion of graphite by hydrogen ions. Nucl Fusion 36(12):1647, 1996. With corrigendum Nucl. Fusion 37, 1997, p. 897.

[26] G. A. van Swaaij, et al. Carbon transport and escape fraction in a high density plasma beam. J Nucl Mater in press. Online http: //dx.doi.org/10.1016/j.jnucmat.2013.01.132

[27| K. R. Umstadter, R. Doerner, G. R. Tynan. Effects of transient heating events on tungsten plasma-facing materials in a steady-state divertor-plasma environment. Nucl Fusion 51(5):053014, 2011.

[28] K. R. Umstadter, R. P. Doerner, G. Tynan. Effect of bulk temperature on erosion of tungsten plasma-facing components subject to simultaneous deuterium plasma and heat pulses. Phys Scr T138:014047, 2009.

[29] W. A. J. Vijvers, et al. Experimental and theoretical determination of the efficiency of a sub-atmospheric flowing high power cascaded arc hydrogen plasma source. Plasma Sources Sci Technol 19(6):065016, 2010. 